\title{
Los números cuentan. Sub-representación de la obra artística de mujeres creadoras en museos y centros de arte contemporáneos
}

\author{
Esmeralda Ballesteros Doncel \\ Universidad Complutense de Madrid \\ eballest@ucm.es
}

Recibido: 22-10-2014

Aceptado: 02-02-2016

\section{Resumen}

Este artículo cuantifica la presencia de obra artística de mujeres artistas en 21 museos y centros de arte contemporáneo españoles. Los resultados constatan una nítida sub-representación de la obra exhibida, por debajo del 20 por ciento. ¿Por qué sucede esto?, ¿diferencial potencial artístico de mujeres y hombres?, ¿superioridad masculina?, ¿discriminación? o ¿un sistema de arte con sesgo androcéntrico? En estas páginas se discute sobre la presencia de varios factores para explicar la brecha de género y se reclama, de las administraciones públicas y las instituciones de gestión cultural, el cumplimiento de la Ley para la Igualdad para garantizar la paridad.

Palabras clave: arte y género; mujeres en las artes visuales; estadísticas de mujeres artistas; desigualdad de género; discriminación de género.

\section{Statistics are Important. The Under-representation of Women in Museum Collections and Art Exhibitions}

\begin{abstract}
This paper analyzes the presence of women artists at the Modern Museums and Art Centers in Spain. The results show an under-representation of women's work in art exhibitions, fewer than $20 \%$. What's the matter? An issue of quality?, Male superiority?, Discrimination?, Androcentric Art System? This article indicate some factor explained the great gap between the genders and demanding to public administrations and cultural institutions the observance of Equality Act 3/2007 to ensure parity.

Key words: art and gender; women in the visual arts; statistics on women artists; inequality gender; discrimination by gender.

\section{Referencia normalizada}

Ballesteros Doncel, E. (2016): "Los números cuentan. Sub-representación de la obra artística de mujeres creadoras en museos y centros de arte contemporáneos", Política y Sociedad, 53 (2), pp. 577-602.

Sumario: Introducción. 1. Metodología. 2. Sub-representación de la obra artística de mujeres creadoras en museos y centros de arte contemporáneos. 3. Pervivencia del sesgo androcéntrico en el sistema de arte en España: hipótesis explicativas. 4. ¿Cuánto tiempo tienen que esperar las mujeres para romper el dominio masculino en el sistema del arte? Estrategias de resistencia y empoderamiento. 5. A modo de conclusión. 6. Bibliografía. 7. Anexos.
\end{abstract}




\section{Introducción}

Perviven grandes mitos en torno al balance de la igualdad de oportunidades entre mujeres y hombres. En el imaginario social se presume que las discriminaciones por razón de género están desapareciendo y, sin embargo, los diagnósticos empíricos siguen descubriendo, una y otra vez, que la igualdad formal difiere de la igualdad real. Identificar los límites que encuentran las mujeres en su desempeño profesional sea en la política, la economía o el arte, ubica esta reflexión en el enfoque de la segregación y la discriminación por razón de género.

Este artículo tiene por objeto principal presentar una estimación cuantitativa sobre la relación de la obra artística, de mujeres y hombres, exhibida en museos de arte contemporáneos en España ${ }^{1}$. En la primera parte se presenta una monitorización estadística de las piezas expuestas en centros de arte. Los datos recabados constituyen una aportación inédita, elaborada a partir de la consulta de una muestra de centros destinados a la exposición, permanente o temporal, de Artes Visuales. En segundo lugar, se señalan los potenciales mecanismos de reproducción de un 'sistema de arte' construido con sesgo androcéntrico, sondeando las mediaciones y prácticas, tanto institucionales como simbólicas, que favorecen la persistencia de desigualdades. El último apartado, describe de forma sucinta las acciones vindicativas de asociaciones feministas que reúnen a creadoras, gestoras y técnicas del arte y que reclaman la urgencia por implementar las directrices marcadas por la Ley 3/2007 -para la igualdad efectiva de mujeres y hombres-, encaminadas a construir escenarios de paridad.

\section{Metodología}

En el marco de una investigación colectiva, que contemplaba obtener un diagnóstico cuantitativo de la presencia de mujeres y hombres en las Artes Visuales, y habida cuenta de que no existe ninguna estadística centralizada que informe sobre la distribución por sexos en este ámbito de actividad económica en España ${ }^{2}$, se diseñó un plan de análisis que incluía cinco dimensiones: 1) Formación-Profesionalización, 2) Actividad económica, 3) Visibilidad-Difusión, 4) Redes profesionales y 5) Gestión ${ }^{3}$. En este artículo se exponen los resultados relativos a la exhibición de la obra artística de mujeres y hombres en museos y centros de arte contemporáneos -visibilidad/difusión-.

${ }^{1}$ Los datos de esta publicación constituyen una parte de los resultados obtenidos en un proyecto de investigación colectivo: «Mujeres y hombres en la industria cultural española: Literatura y Arte Visual», financiado por el Ministerio de Ciencia e Innovación (CICYT: Ref. FEM 2010-16541) y desarrollado bajo la dirección de Fátima Arranz (UCM).

${ }^{2}$ Un ejemplo, en el año 2012, la Subdirección General de Museos Estatales, encargó un informe, basado en la investigación social por encuesta, para conocer la 'situación laboral y formativa' de los profesionales de museos en España. Sorprendentemente, el estudio ya publicado y accesible dese la Web ministerial, no ofrece datos desagregados por sexo, una ausencia más que elocuente y una deficiencia nada inocente, MECD (2012).

${ }^{3}$ En el anexo de este artículo (Tabla 5) se detalla el conjunto de indicadores elaborados y que próximamente serán publicados. 
El ámbito temporal de la cuantificación se circunscribe a los años 2006-2011 y el ámbito geográfico toma como objeto de observación el estado español.

La ausencia de datos cuantitativos publicados, sobre la distribución por sexos de la obra artística de mujeres y hombres en museos y centros de arte contemporáneos, implicó una tarea de compilación estadística minuciosa y rigurosa. Para alcanzar dicho objetivo se diseñó una muestra que incluyera, para cada Comunidad Autónoma, los centros de exhibición de arte contemporáneo de mayor reconocimiento cultural (tabla $1)^{4}$. La muestra contempla 21 espacios expositivos de toda la geografía española, con la excepción de Aragón, Cantabria, Castilla La Mancha y La Rioja, que no cuentan con museos de referencia. En su gran mayoría estas instituciones mantienen una titularidad pública y son de reciente creación (1986-2007). Nacieron con la vocación de difundir el arte de vanguardia, por lo que 'a priori' deberían reflejar una relación equilibrada en la exposición de obra artística de mujeres y hombres.

Este ejercicio comenzó con la búsqueda de dossiers de patrimonio artístico aunque, por su escasez o inexistencia, se procedió a producir una estadística inédita, confeccionada mediante la consulta de las websites de los centros seleccionados. Una actividad laboriosa que implicó un gran número de horas de trabajo y que exigió la contratación temporal de dos ayudantes de investigación ${ }^{5}$.

Las estadísticas obtenidas describen el porcentaje de obra exhibida, según sexo, diferenciando entre: a) colección permanente de los museos, b) exposiciones temporales individuales y c) exposiciones de carácter colectivo. Los datos reunidos permiten, también, diferenciar no sólo entre la obra de varones y mujeres, sino también según la nacionalidad de las artistas -españolas y extranjeras- ${ }^{6}$.

${ }^{4}$ La selección establecida fue confirmada por una crítica de arte, profesora Titular de Estética y Teoría del Arte (Universidad Autónoma de Madrid) y colaboradora del proyecto de investigación FEM 2010-16541: Rocío de la Villa Ardura.

${ }^{5}$ Lucas Olmedo y Fátima Magro, estudiantes de post-grado, fueron las personas encargadas de realizar la mayor parte del trabajo de campo.

${ }^{6}$ Lamentablemente, el diseño de investigación no tuvo en cuenta la disciplina artística dibujo, pintura, grabado, fotografía, video-arte, etc.-, por lo que los resultados están referidos al conjunto de obras expuestas. 
Tabla 1: Muestra de museos y centros ${ }^{7}$ de arte contemporáneos que componen la muestra del estudio, según Comunidades Autónomas y año de inauguración

\begin{tabular}{|c|c|c|c|}
\hline \multirow{2}{*}{ Comunidad Autónoma } & \multicolumn{2}{|c|}{ Museos - Centros de Arte } & \multirow{2}{*}{$\begin{array}{c}\text { Año } \\
\text { de inauguración }\end{array}$} \\
\hline & Centro & Localidad & \\
\hline \multirow{2}{*}{ Andalucía } & CAAC & Sevilla & 1990 \\
\hline & $\mathrm{CAC}$ & Málaga & 2003 \\
\hline Aragón & \multicolumn{3}{|c|}{ No existe museo de arte contemporáneo de referencia } \\
\hline Principado de Asturias & LABORAL & Gijón & 2007 \\
\hline Illes Balears & ESBALUARD & P. de Mallorca & 2004 \\
\hline \multirow{2}{*}{ Canarias } & TEA & St. C. Tenerife & 2008 \\
\hline & CAAM & Las Palmas & 1989 \\
\hline Cantabria & \multicolumn{3}{|c|}{ No existe museo de arte contemporáneo de referencia } \\
\hline \multirow{4}{*}{ Castilla y León } & $\mathrm{Da} 2$ & Salamanca & 2002 \\
\hline & MUSAC & León & 2005 \\
\hline & MEV & Segovia & 1998 \\
\hline & $\mathrm{PH}$ & Valladolid & 2002 \\
\hline Castilla-La Mancha & \multicolumn{3}{|c|}{ No existe museo de arte contemporáneo de referencia } \\
\hline Cataluña & MACBA & Barcelona & 1995 \\
\hline \multirow{2}{*}{ Comunidad Valenciana } & IVAM & Valencia & 1989 \\
\hline & EACC & Castellón & 1999 \\
\hline Extremadura & MEIAC & Badajoz & 1995 \\
\hline \multirow{2}{*}{ Galicia } & MARCO & Vigo & 2002 \\
\hline & CGAV & S. Compostela & 1993 \\
\hline Comunidad de Madrid & MNCARS & Madrid & 1986 \\
\hline Comunidad F. de Navarra & $\mathrm{CACH}$ & Huarte & 2007 \\
\hline \multirow{3}{*}{ País Vasco } & Guggenheim & Bilbao & 1997 \\
\hline & ARTIUM & Vitoria-Gasteiz & 2002 \\
\hline & CCKM & San Sebastián & 1994 \\
\hline La Rioja & \multicolumn{3}{|c|}{ No existe museo de arte contemporáneo de referencia } \\
\hline
\end{tabular}

Fuente: Elaboración propia.

${ }^{7}$ La denominación completa de cada centro de exhibición puede consultarse en el anexo (Tabla 6). 


\section{Sub-representación de la obra artística de mujeres creadoras en museos y centros de arte contemporáneos}

La incorporación al empleo de las mujeres estimuló, en el ámbito de las Ciencias Sociales, numerosas investigaciones desde la perspectiva de género, que fueron señalando de manera generalizada una desigual distribución por sexos en los mercados de trabajo -segregación horizontal y vertical- para, posteriormente, identificar qué restricciones o barreras producían ese asimétrico balance (Jeanes et. al., 2011: xi-Xv). De manera que los ejercicios de cuantificación, como el que aquí se propone, se convierten en una suerte de estribo para iniciar análisis en profundidad que permitan, en última instancia, intervenir y subvertir las desigualdades evidenciadas.

El ámbito de la 'industria cultural' ha sido, hasta el momento, poco explorado desde el marco de la sub-representación por lo que este artículo se presenta como una contribución modesta pero necesaria para visibilizar, una vez más, la persistencia de brechas entre mujeres y hombres.

Tabla 2: Porcentaje de obra artística femenina expuesta en la muestra de museos ${ }^{8}$ y centros de arte contemporáneos, según el carácter de la exhibición, 2006-2011

\begin{tabular}{|l|r|r|r|r|r|r|}
\hline \multirow{2}{*}{ Museo } & \multicolumn{2}{|c|}{$\begin{array}{c}\text { Colección } \\
\text { Permanente }\end{array}$} & \multicolumn{2}{c|}{$\begin{array}{c}\text { Exposiciones } \\
\text { Individuales }\end{array}$} & \multicolumn{2}{c|}{$\begin{array}{c}\text { Exposiciones } \\
\text { Colectivas }\end{array}$} \\
\cline { 2 - 7 } & \multicolumn{7}{|c|}{ Porcentaje de obra artística } \\
\cline { 2 - 7 } & Mujeres & Españolas & Mujeres & Españolas & Mujeres & Españolas \\
\hline MNCARS & 12,7 & 3,7 & 26,8 & 6,6 & 21,2 & 7,3 \\
\hline CAAM & 17,4 & 12,5 & 20,1 & 15,8 & 27,6 & 14,0 \\
\hline IVAM & 9,2 & 5,9 & 13,7 & 6,4 & 19,4 & 5,8 \\
\hline CAAC & 19,8 & 13,0 & 34,1 & 12,8 & 25,5 & 5,4 \\
\hline Thyssen & 2,0 & 0,0 & 5,9 & 0,0 & 4,8 & 0,0 \\
\hline CGAV & 25,8 & 13,0 & 41,9 & 22,6 & 29,4 & 6,2 \\
\hline CCKM & - & - & - & - & 31,2 & 19,0 \\
\hline MACBA & 18,4 & 8,4 & 29,4 & 2,9 & 22,6 & 2,4 \\
\hline MEIAC & 18,7 & 6,8 & 0,0 & 0,0 & 24,3 & 15,1 \\
\hline Guggenheim & 13,7 & 7,6 & 0,0 & 0,0 & 12,5 & 1,7 \\
\hline MEV & - & - & 12,5 & 12,5 & 23,4 & 12,6 \\
\hline EACC & - & - & 25,0 & 0,0 & 47,1 & 8,3 \\
\hline
\end{tabular}

${ }^{8}$ La relación de centros aparece ordenada según la antigüedad del museo-orden descendente-. 


\begin{tabular}{|c|c|c|c|c|c|c|}
\hline \multirow{3}{*}{ Museo } & \multicolumn{2}{|c|}{$\begin{array}{l}\text { Colección } \\
\text { Permanente }\end{array}$} & \multicolumn{2}{|c|}{$\begin{array}{l}\text { Exposiciones } \\
\text { Individuales }\end{array}$} & \multicolumn{2}{|c|}{$\begin{array}{l}\text { Exposiciones } \\
\text { Colectivas }\end{array}$} \\
\hline & \multicolumn{6}{|c|}{ Porcentaje de obra artística } \\
\hline & Mujeres & Españolas & Mujeres & Españolas & Mujeres & Españolas \\
\hline $\mathrm{Da} 2$ & 30,1 & 15,8 & 14,8 & 11,1 & 32,2 & 14,1 \\
\hline PH & 14,6 & 12,7 & 25,7 & 19,3 & 30,4 & 25,7 \\
\hline MARCO & - & - & 24,0 & 16,0 & 27,7 & 7,5 \\
\hline ARTIUM & 21,7 & 17,2 & 20,5 & 5,1 & 18,4 & 10,8 \\
\hline CAC & - & - & 24,6 & 6,1 & - & - \\
\hline ESBALUARD & 12,2 & 7,0 & 10,5 & 7,9 & 14,6 & 6,6 \\
\hline MUSAC & 26,8 & 14,1 & 38,2 & 14,7 & 32,5 & 4,9 \\
\hline LABORAL & - & - & 49,9 & 31,2 & 32,3 & 14,3 \\
\hline $\mathrm{CACH}$ & - & - & 42,4 & 39,4 & 28,5 & 15,9 \\
\hline TEA & 21,9 & 9,0 & 16,6 & 5,5 & 24,8 & 2,7 \\
\hline Promedio & 18,8 & 10,5 & 23,5 & 11,8 & 26,3 & 10,0 \\
\hline Mediana & 18,5 & 10,6 & 24,3 & 9,5 & 26,5 & 7,9 \\
\hline $\begin{array}{l}\text { Desviación } \\
\text { típica }\end{array}$ & 6,1 & 4,1 & 13,4 & 10,4 & 7,6 & 6,2 \\
\hline
\end{tabular}

Fuente: Resultados Proyecto FEM 2010-16541.

Si bien, las producciones y prácticas artísticas de las personas creadoras han adquirido en los últimos años una gran diversidad y dinamismo en sus manifestaciones ${ }^{9}$, lo cierto es que los 'museos' siguen estando considerados por la ciudadanía como los circuitos formales donde se exhibe el 'arte legítimo' (Bourdieu, 1979) o, se manifiesta el esencialismo de la genialidad (Becker, 1982) y, es por ello, que son objeto de visitas multitudinarias y crecientes (AAM, 2012: 1; MNCARS, 2014: 211). De manera que, las obras que se muestran adquieren unos potenciales de difusión masiva que acabará asociándose con el 'caché' artístico sus creadores.

En la tabla 2 se presentan los resultados de cuantificar el porcentaje de obra artística femenina para la muestra seleccionada, según el carácter de la exhibición -colección

${ }^{9}$ La Performance o el Graffiti, por ejemplo, se han consolidado como disciplinas disidentes de los circuitos convencionales de reconocimiento. Sin olvidar el arte no objetual -Body art o Land art- que no es técnicamente museable. 
permanente, exposiciones de carácter temporal (individual y colectiva)-, e identificando, dentro de las piezas creadas por mujeres, las que están firmadas por artistas españolas.

Varias cuestiones llaman la atención. En primer lugar y examinando los valores de las medidas resumen de tendencia central -promedio y mediana- se aprecia que, en todos los centros que componen la muestra, el porcentaje de obra femenina está muy por debajo del umbral de paridad, siendo la obra de las artistas españolas la más sub-representada.

A modo de infografía se propone el gráfico 1, en el que se combina la representación de los promedios, según decilas, asociados al término político 'paridad' ${ }^{10}$. De tal manera que, una relación equilibrada entre los sexos se situará en el intervalo flexible de 40-60 por ciento. La sub-representación se identifica con una participación inferior al 40 por ciento y la sobre-representación reflejaría el desequilibrio inverso (más del 60 \%).

Gráfico 1: Porcentaje de obra artística femenina expuesta en la muestra de museos y centros de arte contemporáneos, según el carácter de la exhibición, 2006-2011

\begin{tabular}{|c|c|c|c|c|c|c|c|c|c|c|}
\hline \multirow{2}{*}{$\begin{array}{l}\text { \% Obra } \\
\text { exhibida }\end{array}$} & \multicolumn{4}{|c|}{ SUB-REPRESENTACIÓN } & PAR & DAD & \multicolumn{4}{|c|}{ SOBRE-REPRESENTACIÓN } \\
\hline & $-10 \%$ & $10-19 \%$ & $20-29 \%$ & $30-39 \%$ & $40-49 \%$ & $50-59 \%$ & $60-69 \%$ & $70-79 \%$ & $80-89 \%$ & $+90 \%$ \\
\hline \multicolumn{11}{|c|}{ COLECCIONES PERMANENTES } \\
\hline $\begin{array}{l}\text { A. Mujeres } \\
\text { A. Españolas }\end{array}$ & 우 & 오 & & & & & & & & \\
\hline \multicolumn{11}{|c|}{ EXPOSICIONES INDIVIDUALES } \\
\hline $\begin{array}{l}\text { A. Mujeres } \\
\text { A. Españolas }\end{array}$ & & 우 & 오 & & & & & & & \\
\hline \multicolumn{11}{|c|}{ EXPOSICIONES COLECTIVAS } \\
\hline $\begin{array}{l}\text { A. Mujeres } \\
\text { A. Españolas }\end{array}$ & 우 & & 오 & & & & & & & \\
\hline
\end{tabular}

Fuente: Resultados Proyecto FEM 2010-16541. Elaboración propia.

El promedio de obras producidas por mujeres (considerando la suma de artistas españolas y extranjeras) en las colecciones permanentes no alcanza al 20 por ciento $(18,8 \%)$. Un desequilibrio que se intensifica si se contabilizan sólo las obras firmadas por artistas de nacionalidad española, donde las piezas adquiridas por los museos sólo representan un

${ }^{10}$ El movimiento por la paridad, iniciado en Francia en los años noventa del siglo XX, ha propiciado no sólo un fructífero debate conceptual (Fraser, 2012: 275-279), sino también novaciones legislativas que en todo el mundo pretenden romper las desigualdades de género -Declaración de Atenas (1992); Resolución de Naciones Unidas A/RES/66/130 (2011)-. 
10,5 por ciento. Esta dimensión es muy importante, en tanto que el patrimonio artístico de cada centro, constituye el fondo con mayor potencial de visibilidad, puesto que se expone de manera continua y su adquisición revierte de manera directa y positiva en la valoración profesional de las personas creadoras.

Imagen 1: Cartel activista ${ }^{11}$

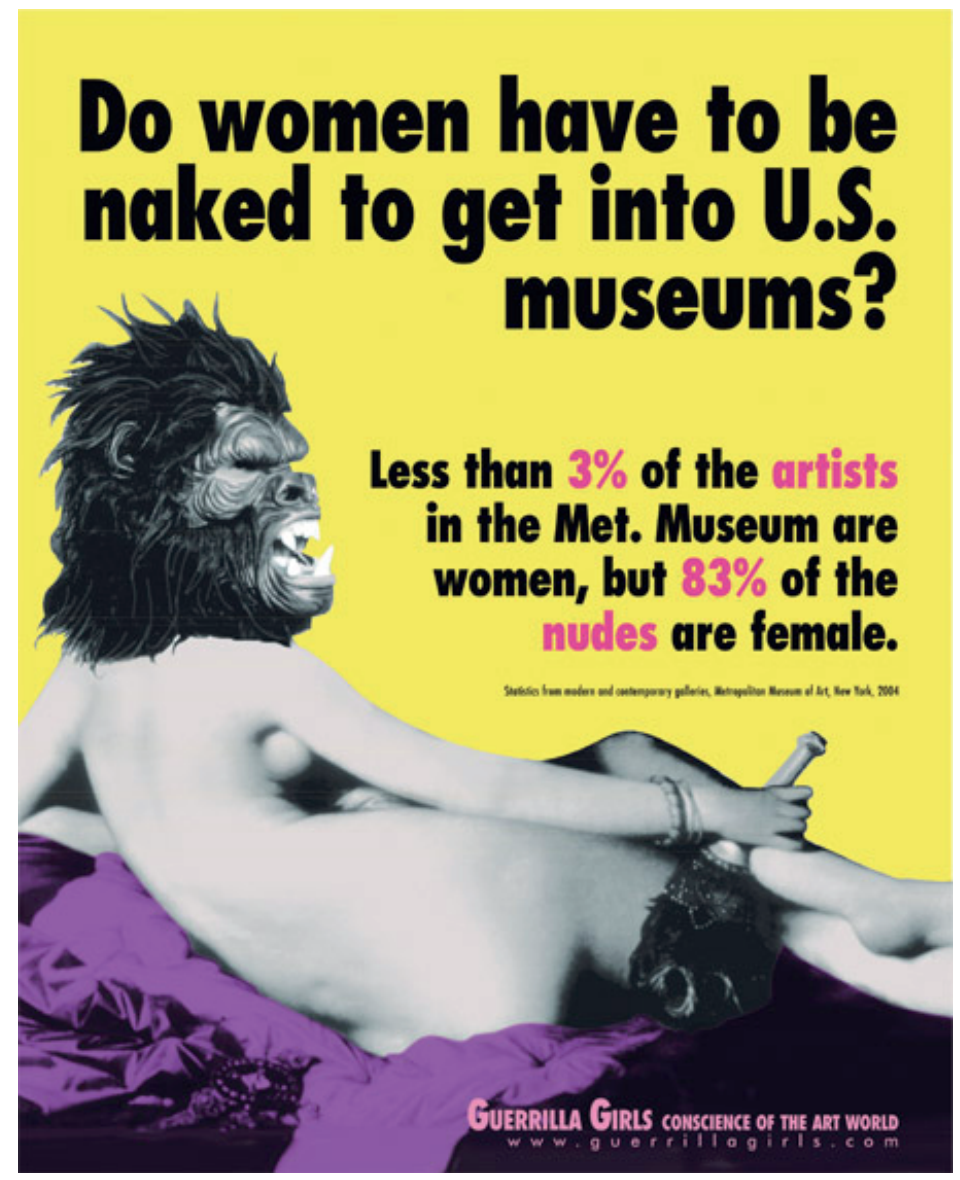

Fuente: Website "Guerrila Girls”, http://www.guerrillagirls.com/, recuperado en abril de 2011.

En lo relativo a exposiciones individuales la información compilada señala que, el porcentaje de artistas mujeres que exhibieron sus creaciones en el período 2006-2011 alcanzó un 23,5 por ciento. Cifra que se reduce a la mitad cuando está referida a las

${ }^{11}$ Cartel activista diseñado, en 1985, como una acción de protesta ante el Museum of Modern Art de Nueva York (MoMa). 
artistas españolas $(11,8 \%)$. Por último, en cuanto a la participación de mujeres en exposiciones colectivas, en el mismo período, el porcentaje de obra femenina se eleva hasta un 26,3 por ciento, mientras que el promedio en el caso de las artistas españolas se sitúa en el diez por ciento.

Estos datos describen un balance de nítida sub-representación que para la Asociación de Mujeres en las Artes Visuales -MAV- proyecta un sistema del arte "atenazado por la discriminación sexista y la falta de igualdad de oportunidades" (MAV, 2012: 3-8).

Tal y como denunciaban irónicamente, las Guerrilla Girls hace tres décadas (imagen 1), la mayor probabilidad de las mujeres para ocupar espacios museísticos guarda relación con ser, o bien 'sujetos - objetos' de inspiración de las obras - deseo o contemplación-, o bien por ser visitantes de los centros (López Fernández Cao, 2000: 23).

Este frustrante diagnóstico no es un fenómeno exclusivo en España. En Francia, los effarantes rapports de Reine Prat en 2006 y 2009, financiados por el Ministerio de Cultura, desvelaron un similar balance de sub-representación que puso 'en pie de guerra' a diversos colectivos, sindicatos y asociaciones, para reivindicar un cambio inaplazable en las políticas públicas de gestión de la cultura desde la perspectiva de género (Gonthier-Maurin, 2013: 5). En el mismo sentido, la crítica Maura Reilly, comisaria junto a la mítica Linda Nochlin de la exposición 'Global Feminisms' -Brooklyn Museum-, publicó recientemente en ArtNews una evaluación de las estadísticas disponibles, confirmando la persistencia de importantes asimetrías de género. Por ejemplo, el porcentaje de las exposiciones individuales dedicadas a artistas mujeres en cinco de los grandes museos americanos -MoMa, Whitney, Guggenheim, MOCA LA ${ }^{12}$, LACMA $^{13}$ - se sitúa en promedio en el 20 por ciento (Reilly, 2015a) ${ }^{14}$.

Este ejercicio de cuantificación no ofrece ninguna primicia sobre el fondo del problema, pues una gran mayoría de mujeres siguen sin acceder al pleno derecho de la libertad (Camps, 2008: 180-182; Torrent, 2012: 199). Pero si, en cambio, objetiva la persistencia de la retórica de la igualdad, pues las mujeres siendo sujetos de los mismos derechos universales, no ven realizadas sus aspiraciones profesionales. Los datos presentados, relativos al período 2006-2011, informan que la obra de las artistas tiene una marginal probabilidad de ser exhibida en los espacios formales-institucionales del arte en nuestro país, especialmente si son españolas. El hecho de que sean estas las más desfavorecidas hace pensar, como señalaron Cabello y Carceller (1998: 230) que el reconocimiento exterior puede ser una mediación para la oportunidad de exhibición en España.

${ }^{12}$ The Museum of Contemporary Art, Los Angeles.

${ }^{13}$ Los Angeles County Museum of Art

${ }^{14}$ Una iniciativa similar se realizó en Londres en el año 2012. Great East London Art Audit llevó a cabo una auditoria sobre la representación de las mujeres en el sistema del arte londinense, descubriendo una evidente desigualdad. Sobre la información proporcionada por 134 galerías comerciales, en las que se representaba a 3.163 artistas, sólo un tercio de las personas representadas eran mujeres. De las 100 subastas más importantes celebradas en 2012, no estaba ninguna pieza realizada por creadoras. Los resultados están disponibles en la siguiente dirección URL http:/elf-audit.com/the-results/ 
De la tabla 2 también se desprende que, los promedios ilustrados por el gráfico 1 encubren una amplia heterogeneidad de escenarios. Parece advertirse un patrón de centros más comprometidos con la paridad -Da2, MUSAC, ARTIUM o CGAV-frente a museos alejados del centro de gravedad de la muestra -MNCARS, Guggenheim, ESBALUARD o IVAM-.

Imagen 2: Cartel activista ${ }^{15}$
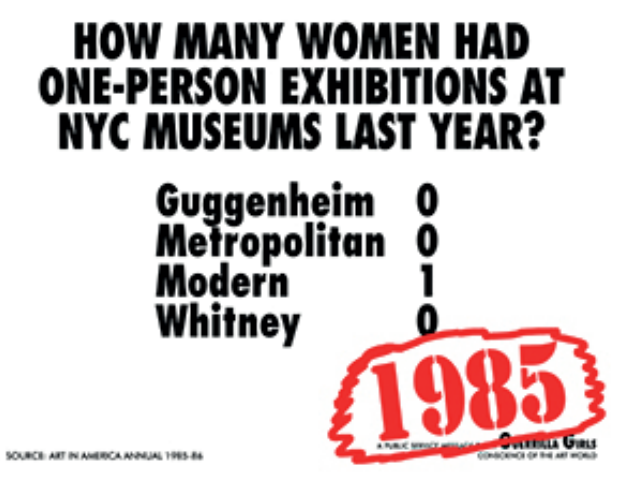

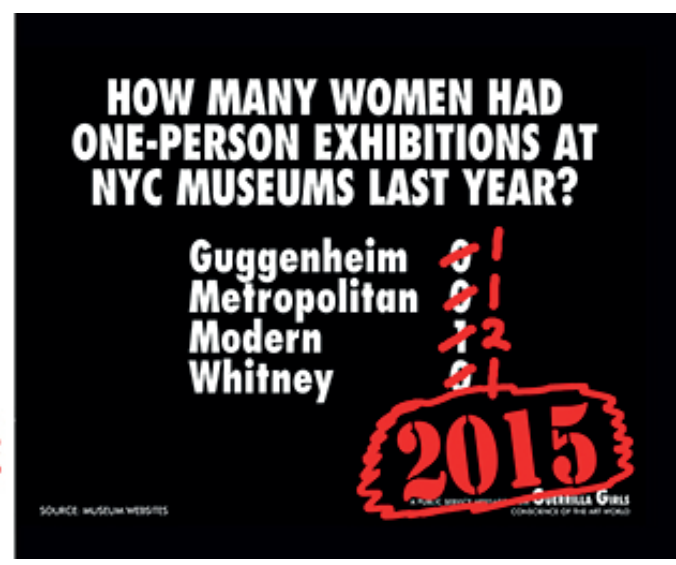

Fuente: Website "Guerrila Girls”, http://www.guerrillagirls.com/, recuperado en noviembre de 2015.

Hasta ahora se ha tratado a las unidades de análisis como similares y, en realidad, las políticas de exhibición de los museos y centros de arte no pueden ser estudiadas sin tener en cuenta variables tan importantes como el presupuesto anual -un indicador consistente de su estatus en el sistema del arte- , la procedencia de la obra patrimonial - colecciones privadas donadas o piezas adquiridas- o la estadística anual de visitantes. Dado que el plan de cuantificación no tuvo en cuenta la consideración de estos datos se realizarán sólo algunos comentarios selectivos.

Probablemente, el Museo Reina Sofía es la institución de arte contemporáneo en España con mayor proyección internacional, no sólo por el presupuesto económico que maneja -varias decenas de millones de euros-año-, sino porque posee un patrimonio aproximado de 30.000 obras y es líder en la estadística de visitantes (TEA-AECOM, 2014: 60-61). Bien pues, según los datos obtenidos en esta cuantificación, también debe señalarse como uno de los espacios de exhibición con mayor sub-representación de obra artística creada por mujeres y de forma particular, de las artistas españolas. Una característica que también ostenta el museo Guggenheim de Bilbao que, al igual que el de Nueva York, ofrece escasas oportunidades a las mujeres (Reilly, 2015a). Estas

${ }^{15}$ Cartel activista diseñado, en 2015, como protesta ante el inmovilismo del sistema del arte, tres décadas después de la primera acción de las Guerrilla Girls. 
informaciones sugieren, como mínimo, reflexionar sobre la brecha de género en el sistema del arte español, para poder en un futuro desarrollar propuestas que rompan con el dominio masculino.

\section{Pervivencia del sesgo androcéntrico en el sistema del arte en España: hipótesis explicativas}

¿Por qué son tan asimétricas las oportunidades de exhibición de la obra artística de mujeres y hombres? Aunque, la investigación empírica desarrollada no tenía por objeto identificar las barreras u obstáculos que producen este balance de desigualdad, pues hubiera requerido otro diseño metodológico, parece necesario enunciar de manera tentativa algunas hipótesis al respecto. Convendría empezar por plantear ¿cómo llega la obra a los museos?, ¿quién o quienes deciden su compra o exhibición temporal?, ¿qué criterios rigen las políticas de adquisición y exhibición de los espacios museísticos?

La existencia de la desigual presencia entre mujeres y hombres en el sistema del arte admite diversas interpretaciones, no coincidentes: a) Culpar a las mujeres -ellas tienen una menor capacidad artística-, b) Atribuirlo a la inercia de la secular división sexual de roles y esperar a que el devenir de la historia rebaje la brecha de género o, c) Imputar las desigualdades a la construcción social de un sistema del arte con sesgo androcéntrico. En las siguientes líneas se apuntan algunas líneas de trabajo para la discusión.

\subsection{Diferencial potencial artístico de mujeres y hombres}

Existe un discurso convencional, cuestionado pero no extinguido, que apela a la posesión del ingenio como una cualidad abstracta y, por tanto, sin sesgo de género que conduciría a afirmar que, si la obra de las artistas no ocupa un lugar preeminente en los centros de arte contemporáneos, es porque 'ellas tienen una menor capacidad'. Esta opinión ha sido profusamente debatida y desafiada.

Cuando en 1971, Linda Nochlin, preguntaba irónica e insistentemente "Why Have There Been No Great Women Artists" estaba abriendo la caja de pandora al activismo de las mujeres creadoras. Su artículo cuestionaba el orden masculino, resaltando su significado como un dominio construido socialmente y sus conclusiones advertían de que el arte no es una actividad libre y autónoma de un individuo con capacidades innatas, sino el resultado de la estructura social, mediada y determinada por instituciones, ya sean academias de arte, sistemas de patronazgo o, mitologías de lo divino. Su desafío fue seguido por centenares de profesionales que, durante décadas han desarrollado sólidas investigaciones empíricas para cuestionar la hegemonía masculina (Mayayo, 2003: 25-62; Muñoz López, 2012: 395) y recuperar del olvido y la ocultación las prácticas artísticas de grandes creadoras (Torrent, 2012: 203-207). En este sentido es fundamental preguntar(se) ¿cómo se establecen los criterios de reconocimiento que, hasta ahora, han excluido, ignorado o minusvalorado el arte producido por mujeres? 


\subsection{La paridad requiere tiempo}

En segundo lugar, existiría un discurso del conformismo, complementario a la primera interpretación, que invocaría a que las desigualdades advertidas serían el resultado de la inercia secular en la división sexual de roles -masculino/femenino-. Las mujeres artistas serían minoría y sus producciones escasas, por lo que ellas aún necesitarían más tiempo para desarrollar carreras de éxito y para que la igualdad de género sea, en el futuro, una realidad ${ }^{16}$. No obstante, este argumento puede desmontarse si se considera que desde hace varias décadas las mujeres son mayoría en los espacios de formación reglada (tabla 3 ).

Tabla 3: Evolución de la matrícula en las licenciaturas de Bellas Artes e Historia del Arte, 1994-2010

\begin{tabular}{|c|c|c|c|c|}
\hline \multirow{2}{*}{$\begin{array}{l}\text { Curso } \\
\text { académico }\end{array}$} & \multicolumn{2}{|c|}{ Bellas Artes } & \multicolumn{2}{|c|}{ Historia del Arte } \\
\hline & $\begin{array}{l}\text { Alumnado } \\
\text { Matriculado }\end{array}$ & $\begin{array}{c}\% \\
\text { Mujeres }\end{array}$ & $\begin{array}{l}\text { Alumnado } \\
\text { Matriculado }\end{array}$ & $\begin{array}{c}\% \\
\text { Mujeres }\end{array}$ \\
\hline $1994 / 95$ & 11.128 & 62,41 & 5.177 & 73,56 \\
\hline $1995 / 96$ & 11.975 & 62,30 & 9.048 & 72,53 \\
\hline $1996 / 97$ & 12.217 & 62,36 & 12.504 & 73,12 \\
\hline $1997 / 98$ & 12.188 & 62,13 & 14.757 & 72,68 \\
\hline 1998-1999 & 12.106 & 61,69 & 16.512 & 73,08 \\
\hline 1999-2000 & 12.461 & 61,67 & 17.293 & 72,79 \\
\hline $2000-2001$ & 12.583 & 62,90 & 16.916 & 73,94 \\
\hline 2001-2002 & 12.608 & 62,84 & 16.398 & 73,75 \\
\hline $2002-2003$ & 13.264 & 63,15 & 15.309 & 73,83 \\
\hline 2003-2004 & 13.319 & 64,31 & 14.298 & 74,58 \\
\hline 2004-2005 & 13.478 & 64,84 & 13.451 & 74,25 \\
\hline $2005-2006$ & 13.573 & 65,45 & 12.181 & 74,40 \\
\hline $2006-2007$ & 13.790 & 65,49 & 11.425 & 74,99 \\
\hline $2007-2008$ & 13.562 & 65,21 & 10.652 & 74,09 \\
\hline
\end{tabular}

${ }^{16}$ En este sentido parece oportuno citar la estimación de la novena edición del informe Global Gender Gap -2014- que señala que, la relación entre mujeres y hombres en el mundo tardará, al menos, 80 años en conquistar el umbral de la paridad. 


\begin{tabular}{|l|c|c|c|c|}
\hline \multirow{2}{*}{$\begin{array}{l}\text { Curso } \\
\text { académico }\end{array}$} & \multicolumn{2}{|c|}{ Bellas Artes } & \multicolumn{2}{c|}{ Historia del Arte } \\
\cline { 2 - 5 } & $\begin{array}{c}\text { Alumnado } \\
\text { Matriculado }\end{array}$ & $\begin{array}{c}\% \\
\text { Mujeres }\end{array}$ & $\begin{array}{c}\text { Alumnado } \\
\text { Matriculado }\end{array}$ & $\begin{array}{c}\% \\
\text { Mujeres }\end{array}$ \\
\hline $2008-2009$ & 13.284 & 65,40 & 10.253 & 73,64 \\
\hline $2009-2010$ & 12.670 & 65,33 & 8.126 & 73,48 \\
\hline
\end{tabular}

Fuentes: 1994-1998 (MEC: 2002) y 1998-2010 (MEC -varios años-, Estadística de la Educación, Enseñanzas universitarias: Alumnado. Elaboración propia.

Las estadísticas del Ministerio de Educación confirman, desde principio de los años noventa, una feminización en la matrícula de estudios universitarios vinculados a la formación artística, ya constatada por López de la Cruz (2002: 297-298).

Tabla 4: Evolución del porcentaje de mujeres licenciadas en la UE y España en al área de Humanidades y Artes

\begin{tabular}{|l|l|l|l|l|l|l|l|l|l|}
\hline \multirow{2}{*}{$\begin{array}{l}\text { Ámbito } \\
\text { territorial }\end{array}$} & \multicolumn{10}{|c|}{ Años } \\
\cline { 2 - 11 } & 2008 & 2007 & 2006 & 2005 & 2004 & 2003 & 2002 & 2001 & 2000 \\
\hline España & 61,2 & 61,9 & 62,3 & 62,5 & 63,0 & 62,6 & 61,7 & 62,8 & 62,2 \\
\hline UE 27 & 68,9 & 69,0 & 69,4 & 69,2 & 69,3 & 70,2 & 68,7 & 68,7 & 68,8 \\
\hline
\end{tabular}

Fuente: Eurostat. Elaboración: INE (2012), Estadística de Enseñanza Universitaria.

Si bien, la capacidad creadora no puede restringirse a la posesión de credenciales educativas, son un factor que determina tanto la oferta como la demanda de los mercados de trabajo. La sobre-representación de mujeres licenciadas en el área de Artes es constatable no sólo a escala nacional, sino también en Europa (tabla 4), por lo que se evidencia una notable controversia: siendo mayoría en las aulas de formación no alcanzan un rango de igualdad, con sus pares, en el ámbito de la profesionalización.

Parece oportuno recuperar, a este respecto, la reflexión personal de Patricia Mayayo (2003: 11) en su período formativo:

"Cuando inicié mis estudios universitarios de historia del arte, hacia mediados de la década de 1980, hubo un par de 'detalles' que enseguida llamaron mi atención. Iban pasando los meses (y según pude comprobar posteriormente, los años) y ni un solo nombre de mujer artista aparecía mencionado en el transcurso de las clases o en los manuales más consultados (tan solo al llegar a la historia de las vanguardias históricas empezaron a asomar tímidamente la cabeza algunas creadoras). Tampoco eran muchos los nombres de historiadoras del arte consignados en las bibliografias 
de curso. ¿Acaso no había existido ni una sola mujer artista hasta principios del siglo XX? ¿De verdad eran tan pocas las estudiosas de la historia del arte que habían hecho contribuciones valiosas a la disciplina? ¿Cómo justificar entonces mi presencia en aquellas aulas? ¿Me esperaba también a mí la invisibilidad profesional?”

La posesión de competencias formativas, como capacidades potenciales para la práctica profesional, parece no estar garantizada al género femenino. De modo que, si las credenciales educativas sólo adquieren preeminencia cuando se intercambian en el mercado de trabajo, los títulos académicos no son un aval para el empleo de las artistas, pues el 'capital escolar' sólo adquiere valor si consigue transformarse en 'capital social' (Baudelot y Establet, 1992: 15-18). En otras palabras, el acceso a circuitos públicos de reconocimiento estaría mediado por sutiles barreras que, de forma acumulativa y sinérgica, frenan el acceso de las creadoras al poder de representación.

\subsection{Un sistema museográfico con sesgo androcéntrico}

Desde una perspectiva crítica, este diagnóstico señalaría la responsabilidad de las organizaciones e instituciones del sistema del arte de no ofrecer las mismas oportunidades y favorecer un sistema de orden androcéntrico (Acker, 2006; Fraser, 2007).

Ya se indicó que los museos son percibidos por el imaginario colectivo como los 'templos de la creación humana'. Paseamos por ellos sin conocer la distribución de las piezas según el sexo, la etnia o la diversidad funcional de los artistas y, aunque, el gusto es una dimensión subjetiva, su justificación tiene una naturaleza social (Camps, 2008: 188). Las obras exhibidas tienen el potencial de ser aceptadas como verdades universales por parte de la audiencias que los absorben (Mclean, 1999: 84). Sin embargo, esta idea obvia los condicionamientos socio-políticos de ese proceso, ignorando que el reconocimiento del 'arte legítimo' es, como tantos otros procesos, una construcción social muy afectada por la existencia de redes profesionales, a las que Pierre Bourdieu (1979) y Howard Becker (1982), denominaron campo y sistema, respectivamente. De forma que, la genialidad artística, estaría mediada por unas estructuras profesionales afectadas por posiciones ideológicas, que configuran relaciones en competencia y conflicto entre actores muy diversos -artistas, galeristas, medios de comunicación, críticos, coleccionistas, etc.-

Asumiendo que el análisis de las organizaciones es una cuestión clave para entender las lógicas androcéntricas en el ámbito de las relaciones laborales (Kanter, 1977; Cockburn, 1991) resulta ineludible examinar las características de los órganos de decisión de los espacios museísticos - dirección y patronatos-, pues es en ellos donde se determinan las políticas de gestión, es decir, son el filtro para decidir qué es arte legítimo y qué no, y por tanto, qué tendrá visibilidad y respaldo y qué se quedará fuera.

En el gráfico 2 se describe la composición por sexo en tres ámbitos de decisión y asesoría en el sistema museográfico español: la Junta Superior de Museos, Patronatos y Visitantes; los Patronatos de cada museo o centro de arte contemporáneo y la 
dirección de estos ${ }^{17}$. Los datos recabados, de nuevo, describen un diagnóstico de subrepresentación.

En relación a la Junta Superior de Museos, Patronatos y Visitantes el gráfico muestra una distribución paritaria: 13 hombres (59\%) y 9 mujeres (41\%). Si bien, la Junta, es el máximo órgano consultivo de la administración general del Estado en materia de Cultura y Patrimonio, su función principal es de reglamentación de museos de titularidad estatal y, asesoría a la Dirección General de Bellas Artes y Bienes Culturales, por lo que sus competencias se centran en establecer las directrices generales del sistema museográfico ${ }^{18}$.

Gráfico 2: Participación de mujeres en órganos de dirección y gestión de museos

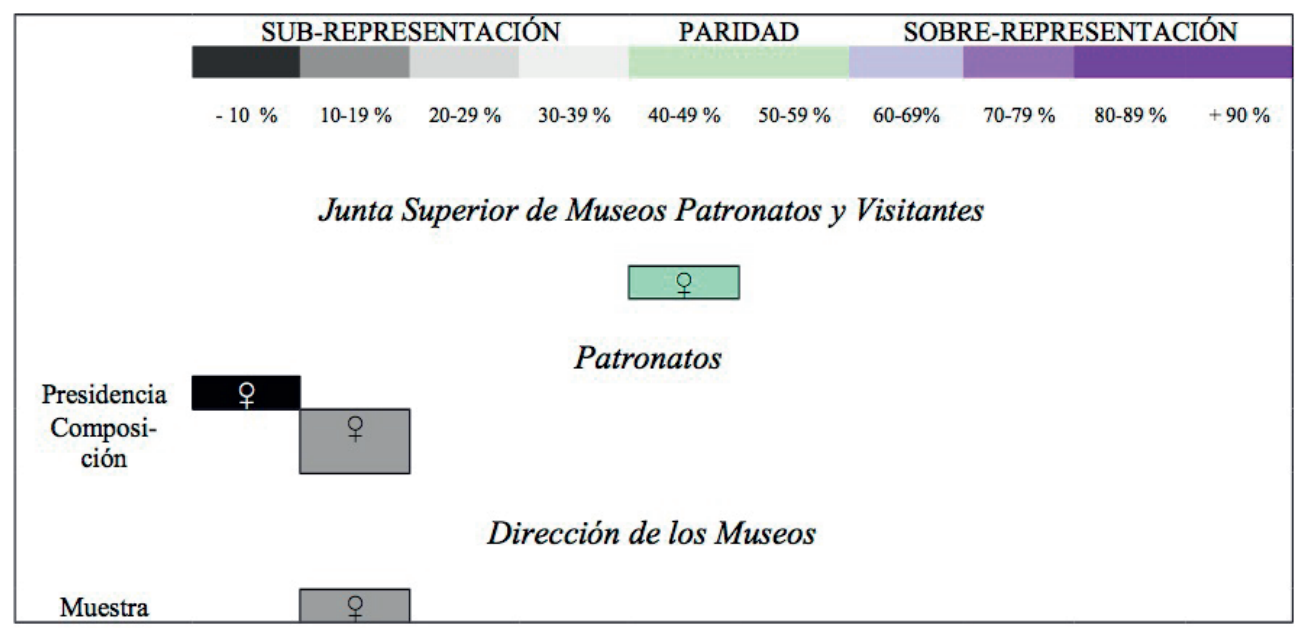

Fuentes: Tablas 6 y 7 de los Anexos. Elaboración propia.

El organismo principal de gobierno de los museos, donde se determinan las políticas de adquisición y exhibición, es el patronato. Este se erige como un consejo colegiado, formado por distintas instituciones de gestión territorial -alto personal de Administraciones Públicas- y, en ocasiones, representantes empresariales. El patronato

${ }^{17}$ Examinar la distribución por sexo en la composición de los órganos de decisión del sistema museístico es sólo una característica de análisis que tan sólo inicia la discusión.

${ }^{18}$ Los miembros de la Junta se establecen por orden ministerial, ocupando la presidencia la persona titular en la DG de Bellas Artes. A ésta, acompañan representantes de museos estatales, del Instituto del Patrimonio Histórico Español, Junta de Calificación, valoración y exportación de Bienes de Patrimonio Histórico Español y un delegado/a por cada Comunidad Autónoma. Orden del 13 de junio de 2001 por la que se determina la composición de la Junta Superior de Museos (Boletín Oficial del Estado, núm. 152 de 26 de junio de 2001, página 22590). 
de cada museo realiza funciones de regulación, control y decisión, entre las que se encuentra la designación de la dirección de los centros de arte.

El gráfico 2 señala que, la presidencia de los patronatos es abrumadoramente masculina, sólo una mujer de los once centros para los que se ha obtenido información. En cuanto a la composición por sexo de los patronatos se han contabilizado 130 hombres $(73,4 \%)$ y 47 mujeres $(26,6 \%)$. Este diagnóstico, es una de las denuncias recurrentes de la Asociación de Mujeres en Artes Visuales (MAV, 2012: 25).

Por último, la dirección de los museos y centros de arte seleccionados también verifica un balance de sub-representación. Cuatro mujeres directoras (18\%) frente a dieciocho hombres (82\%). Estas 'privilegiadas' ostentan su cargo en espacios con unas estadísticas de visitantes moderadas ${ }^{19}$. La directoras están al cargo de centros con un presupuesto anual modesto, una característica observada en norteamérica por la Association of Art Museum Directors (2015:3) y denunciada por profesionales del arte como Nochlin (2007) o Maura Reilly (2015a). Aunque quizá el efecto más pernicioso de la desigualdad sea privar a la sociedad de nuevas formas de concebir e interpretar las programaciones de los centros de exhibición (Blatter, 2014: 68-73).

La sub-representación de las mujeres en las estructuras institucionales de gestión del arte define y redefine su participación en los sistemas artísticos. La falta de igualdad de oportunidades en la exhibición del arte hecho por mujeres implica una silenciación impuesta, entendida por algunas artistas como una forma sutil de violencia de género (Torrent, 2012: 199). La invisivilización de sus narrativas artísticas priva a la sociedad de conocer sus discursos y de disfrutar de sus producciones que, como señalaba Marcela Lagarde, pueden "revolucionar el orden de poderes entre los géneros y con ello la vida cotidiana, las relaciones, los roles y los estatutos de mujeres y hombres" (Lagarde, 1996: 18). Pero en última instancia, mantener a las artistas en el anonimato es privarlas del derecho de la libertad, que teóricamente tienen pero que no ejercen, y perpetuar una democracia incompleta (Camps, 2008: 180-181).

\section{4. ¿Cuánto tiempo tienen que esperar las mujeres para romper el dominio masculino en el sistema del arte? Estrategias de resistencia y empoderamiento}

Señalar los problemas es el principio de su solución, condición necesaria pero no suficiente para promover un cambio sustantivo. Ciertamente, denunciar el desequilibrio de oportunidades en la exhibición de la obra artística de mujeres y hombres debe ser un estímulo para iniciar acciones políticas encaminadas a corregir las desigualdades de género desde ámbitos institucionales y asociativos.

En este punto es necesario plantear si esta asimetría, sobre-representación masculina y sub-representación femenina en el sistema del arte, ha recibido atención pública y ha sido objeto de debate político. Efectivamente, el texto de la Ley 3/2007 para la

${ }^{19}$ Ana Martínez Aguilar (Museo Esteban Vicente), Cristina Fontaneda Berthet (Museo Patio Herreriano), Lorenza Barboni (Espai d'Art Contemporani de Castelló) y Consuelo Císcar (Instituto Valenciano de Arte Moderno). 
Igualdad efectiva de Mujeres y Hombres -en adelante Ley para Igualdad-contempla en su artículo 26, disposiciones específicas de promoción de la equidad en el ámbito de la creación y producción artística. En concreto, en el punto 2 apartado c) señala:

"Las autoridades públicas...desarrollaran las siguientes actuaciones: Promover la presencia equilibrada de mujeres y hombres en la oferta artística y cultural pública".

Este mandato, se inscribe en la declaración de que el pleno reconocimiento de la igualdad formal ante la ley (artículo 14 de la CE de 1978), no se ha traducido en una igualdad real, por lo que son necesarias políticas de acción positiva que favorezcan los desequilibrios advertidos. No obstante, esta prescripción jurídica no parece suficiente como mecanismo efectivo para aminorar la desventaja de las mujeres, en relación a las oportunidades de exhibición de sus obras en museos y centros de arte contemporáneos. Quizá porque, como denuncia la Asociación de Mujeres en las Artes Visuales, este mandato ha sido ignorado por parte de los agentes del sistema del arte en España (MAV, 2012: 5-6). Sin olvidar que, la Ley para la Igualdad ha tenido un escaso desarrollo reglamentario, no ha contado con un presupuesto de acompañamiento y, lo que es peor, no se realiza ningún seguimiento, ni evaluación del alcance de la misma. De modo que la legislación en vigor no procura, por sí misma, la igualdad entre los sexos, si no se establecen dispositivos de evaluación y tutela de sus preceptos (Camps, 2008: 188).

En la última década, la falacia de la igualdad ha provocado la emergencia en España de un activismo feminista en el sistema de arte que expresa la disconformidad con este estado de cosas y exige que se apliquen las directrices de la Ley para la Igualdad, aduciendo que, si las estadísticas no cambian no es por falta de talento o de producción artística por parte de las mujeres, sino por falta de voluntad política.

En España, el asociacionismo feminista en las Artes es muy reciente como lo demuestran las fundaciones de diversas organizaciones con visibilidad mediática en la defensa de los derechos de las mujeres artistas: CIMA -Asociación de Mujeres Cineastas y de Medios Audiovisuales-, MAV-Asociación de Mujeres en Artes Visualesy Clásicas y Modernas -Asociación para la igualdad de género en la cultura. Fundadas entre 2006 y 2009, vienen desarrollando múltiples acciones y propuestas, entre las que sobresale la demanda de cumplimiento de la prescripción del artículo 26.d de la Ley de Igualdad:

"Que se respete y se garantice la representación equilibrada en los distintos órganos consultivos, científicos y de decisión existentes en el organigrama artístico y cultural".

Las estrategias de activismo feminista en España, comienzan por "re"elaborar una historia del arte en el que aparezcan las mujeres para, así, tener referentes y reivindicar la importancia y posición de un número nada despreciable de artistas, mediante artículos, ensayos y libros recogidos en las bibliografías de sus páginas Web.

Este mecanismo de visibilización de las mujeres en el arte está acompañado de otros como la instauración de premios -Premio MAV-, la promoción de exposiciones 
-Genealogías feministas en el arte español: 1960-2010 organizado por el MUSAC y el MNCARS ${ }^{20}$-, la organización de festivales -Festival Miradas de Mujeres-, la elaboración de informes y publicaciones vindicativas, la convocatoria de encuentros, debates, y prácticas de impacto visual-mediático -performances o flash mobs-. Estas acciones se encaminan a proyectar la existencia del arte realizado por mujeres y a denunciar el diagnóstico de marginalidad en el que se encuentran en el sistema institucional.

Conjuntamente con estas acciones de sensibilización se llevan a cabo estrategias de presión sobre organismos públicos para exigir la entrada de las artistas en los órganos de decisión del sistema del arte. De esta manera, las organizaciones antes mencionadas han elaborado manifiestos difundidos en el ámbito profesional -Manifiesto ARCO $2005^{21}$-, o dirigidos a la sociedad en su conjunto -Manifiesto por la igualdad en la Cultura 201322-, han elevado cartas a museos (Acción 'Correos'-2010), Ayuntamientos o al Defensor del Pueblo (2011), articulando una red de acciones y propuestas para subvertir los diagnósticos de desigualdad y anunciar una incesante vigilancia (López Fernández Cao, 2014).

\section{A modo de conclusión}

Considerando que las estadísticas socio-demográficas informan que la población de mujeres y hombres es similar $(50,8 \%-49,1 \%)$, teniendo en cuenta que las mujeres son mayoría en la formación universitaria asociada al ámbito de conocimiento del arte y, sin olvidar que, desde 1978 la Constitución española consagra la igualdad jurídica de la personas (art. 14), el diagnóstico de desigualdad advertido mediante este ejercicio de cuantificación, es una característica injusta, no sólo en España sino en otros países del denominado 'mundo desarrollado'.

Este resultado no es un escenario inevitable, sino el resultado de un 'reparto de reconocimiento' con sesgo androcéntrico, tal y como denuncian las distintas profesionales del sistema del arte -creadoras, técnicas, galeristas, críticas, etc.-. Esta situación puede y debe ser subvertida, mediante el desarrollo de políticas de gestión cultural que tengan en cuenta dos dimensiones: la redistribución y el reconocimiento (Fraser, 2007: 274-275). En primer lugar, la distribución paritaria exige, tal y como prescribe la Ley para la Igualdad -art. 26.c-: "que se promueva la presencia equilibrada de mujeres y hombres en la oferta artística y cultural pública", corrigiendo las desigualdades observadas en las oportunidades de exposición. Y, en segundo lugar, que los criterios de reconocimiento artístico tengan en cuenta una nueva diversidad estética, receptiva no sólo al género, sino también a la etnia, la identidad sexual o la diversidad funcional, pues las diversas categorías sociales, hasta ahora excluidas

${ }^{20}$ MUSAC (2011): Genealogías feministas en el arte español, 1960-2010.

${ }^{21}$ La iniciativa de Xabier Arakistain de promover el Manifiesto Arco 2005, por el que exigía la igualdad en los museos españoles fue una actuación simbólica que no obtuvo ninguna reacción a nivel nacional pero de la que si se hizo eco la prensa internacional (Reilly, 2015a).

${ }^{22}$ Promovido por CyM, CIIMA, AMIT y MAV. 
o minusvaloradas, deben tener el mismo estatus en la interacción social del sistema del arte. Para la consecución de tal fin, se requiere que las profesionales del arte se incorporen, de forma paritaria a los órganos de decisión que diseñan y desarrollan las políticas de adquisición y exhibición en los museos -art. 26.d de la Ley para la igualdad: "Que se respete y se garantice la representación equilibrada en los distintos órganos consultivos, científicos y de decisión existentes en el organigrama artístico y cultural".

La ausencia de mujeres en los espacios de decisión define y redefine la organización de los mundos del arte y sus efectos no se limitan a la invisibilización de sus creaciones y prácticas artísticas, sino que implica no poder negociar sus preocupaciones, amenazas y menosprecios a sus prácticas creadoras. Efectivamente, las mujeres deben estar presentes, en igualdad de condiciones a sus pares varones en los patronatos y la dirección de los centros de arte, confiando en que no sólo se rompa con el dominio masculino, sino que se inicien y promuevan cambios culturales en los patrones de reconocimiento artístico... "que la realidad en la que vivimos sea de todas y todos y no sólo de unos cuantos" (Camps, 2008: 191).

\section{Bibliografía}

AAM -American Alliance of Museums- (2012): "Making the Case about the Value of Museums", Washington. Disponible en:

http://www.aam-us.org/about-museums/facts, fecha de acceso diciembre de 2015.

Acker, J. (2006): "Inequality Regimes: Gender, Class, and Race in Organizations", Gender and Society, 20, pp. 441-464, Disponible en:

http://www.sagepub.com/oswcondensed/study/articles/05/Acker.pdf.

TEA/AECOM (2014): Theme Index and Museum Index: The Global Attractions Attendance Report, ed. Themed Entertainment Association. Disponible en: http://www.teaconnect.org/images/files/TEA_103_49736_150603.pdf, fecha de acceso diciembre de 2015.

Baudelot, Ch. y R. Establet, (1992): Allez, les filles!, París, Editions du Seuil.

Becker, H. S. (1982): Los mundos del arte: sociología del trabajo artístico, Universidad Nacional de Quilmes, Bernal, 2008.

Blatter, S.M. (2014): The Role of American Women in Museum Leadership: Late 19th to Mid-20th Century, Museum Studies Theses, Paper 6. Disponible en:

http://digitalcommons.buffalostate.edu/cgi/viewcontent. cgi?article $=1006 \&$ context $=$ museumstudies_theses.

Bourdieu, P. (1979): La distinción: criterios y bases sociales del gusto", Madrid, Taurus, 1988.

Cabello, H. y A. Carceler (1998): "Mirando hacia dentro. La situación de la obra de las mujeres en el panorama artístico actual", Arte, Individuo y Sociedad, 10, pp. 229244.

Camps, V. (2008): "Las mujeres y el ejercicio de la libertad", en Puleo, A. (coord.), El reto de la igualdad de género: Nuevas perspectivas en ética y filosofia política, Madrid, Biblioteca Nueva, pp. 179-191. 
Clásicas y Modernas (2013): Manifiesto por la Igualdad en la Cultura. Disponible en: http://www.clasicasymodernas.org/wp-content/uploads/CyM-CIIMA-AMIT-MAVMANIFIESTO-POR-LA-IGUALDAD-EN-LA-CULTURA-1-3-2013.pdf.

Cockburn, C. (1991): In the way of women: men's resistance to sex equality in organizations, Basingstoke, Palgrave Macmillan.

Declaración de Atenas/Athens Declaration (1992). Disponible en: http://eurogender.eige.europa.eu/news/athens-declaration-1992.

EUROSTAT (2012): Estadísticas de Enseñanza Universitaria, en INE: http://www.ine.es/jaxi/menu.do?type=pcaxis\&path=/t13/p405\&file=inebase.

Fraser, N. (2007): "Feminist politics in the age of recognition: A two-dimensional approach to gender justice", Studies in Social Justice, 1 (1), pp. 23-35. Traducido por Marta Postigo Asenjo y publicado en la Revista Arenal, "La política feminista en la era del reconocimiento: un enfoque bidimensional de la justicia de género", 2012, 19 (2), pp. 267-286.

Gonthier-Maurin, B. (2013): Rapport d'Information Fait au nom de la délégation aux droits des femmes et à l'égalité des chances entre les hommes et les femmes sur le thème «La place des femmes dans l'art et la culture», Présidence du Sénat en France Disponible en: http://www.senat.fr/notice-rapport/2012/r12-704-notice.html.

Jeanes, E., Knights, D. y P. Yancey (eds.) (2011): Handbook of Gender, Work and Organization, Chichester, West Sussex, Wiley.

Kanter, R. M. (1977): "Some effects of proportions on group life: skewed sex ratios and responses to token women", American Journal of Sociology, 82 (5), pp. 965-990.

Lagarde, M. (1996): Género y feminismo. Desarrollo humano y democracia, Madrid, Ed. Horas y Horas.

López de La Cruz, L. (2001): "La presencia de la Mujer en la Universidad española", Revista Historia de la Educación Latinoamericana, 4, pp. 291-299.

López Fernández Cao, M. (2000): "La creación artística: un difícil sustantivo femenino", en F. Cao (coord.), Creación artística y mujeres. Recuperar la memoria, Madrid, Narcea, pp. 13-47.

López Fernández Cao, M. (2014): “MAV te observa, entraremos en acción: Las mujeres en el sistema del arte español. Sobre piedras y vientos de igualdad", Investigaciones Feministas, 4, págs. 91-110.

Mayayo, P. (2003): Historias de mujeres, historias de arte, Madrid, Cátedra.

McLean, K. (1999): "Museums Exhibitions and the Dynamics of Dialogue." Daedalus - Journal of the American Academy of Arts and Sciences-, 128 (3), pp. 83-107.

MEC (2002): Estadística Universitaria (1994-2001), Madrid, ed. Consejo de Coordinación Universitaria.

MEC (2007): Académicas en Cifras, Madrid, Unidad de Mujeres y Ciencia (UNMYC). MECD (2011): Patrimonio en femenino. Disponible en: http://www.calameo.com/read/0000753353fdf029c796c

MECD (2012): Los profesionales de los museos. Un estudio sobre el sector en España, Madrid, Ed. Secretaria General Técnica del Centro de Publicaciones del MECD. Disponible en: 
https://sede.educacion.gob.es/publiventa/descarga.action?f_codigo_agc=14316C.

MNCARS (2014): Memoria de Actividades del Museo Nacional Centro de Arte Reina Sofia. Disponible en:

http://www.museoreinasofia.es/museo/memoria-actividades.

Mujeres en Artes Visuales -MAV- (2012): La situación de las mujeres en el sistema del arte en España. Propuestas de actuación, disponible en:

http://www.mav.org.es/documentos/DOCUMENTO\%20DE\%20TRABAJO\%20 MAV\%202012.pdf.

Muñoz López, P. (2012): "Las mujeres como creadoras en las artes plásticas según los textos de hombres y mujeres en la España de la primera mitad del siglo xx", Revista Arenal, 19 (2), pp. 393-413.

Nochlin, L. (1971): "Why Have There Been No Great Women Artists?" ARTnews January-, pp. 22-39.

Nochlin, L. (2007): "Where the Great Women Artists are Now", ARTnews -February-. Disponible en:

http://www.artnews.com/2007/02/01/where-the-great-women-artists-are-now/.

Reilly, M. (2015a): “Taking the measure of sexism: Facts, Figures, and Fixes", ARTnews. Disponible en:

http://www.artnews.com/2015/05/26/taking-the-measure-of-sexism-facts-figuresand-fixes/.

Reilly, M. (2015b): "Linda Nochlin on Feminism then and now", ARTnews. Disponible en:

http://www.artnews.com/2015/05/26/linda-nochlin-on-feminism-then-and-now/.

TEA-AECOM (2014): Theme Index and Museum Index: The Global Attractions Attendance Report. Disponible en: http://www.teaconnect.org/images/files/TEA_103_49736_150603.pdf.

Torrent Escaplés, R. (2012): "El silencio como forma de violencia. Historia del arte y mujeres", Arte y políticas de identidad, 6, pp. 199-213.

UN (2011): La participación de la mujer en política -Resolution adopted by the General Assembly (A/RES/66/130): Women and political participation. Disponible en: http://www.un.org/womenwatch/daw/documents/ga66.htm.

World Economic Forum (2014): Global Gender Gap Report 2014. Disponible en: http://www3.weforum.org/docs/GGGR14/GGGR_CompleteReport_2014.pdf. 


\section{Anexos}

Tabla 5: Indicadores producidos en la cuantificación del balance entre mujeres y hombres en las Artes Visuales

\begin{tabular}{|c|c|}
\hline Indicadores & Dimensión y descripción \\
\hline \multicolumn{2}{|c|}{ FORMACIÓN-PROFESIONALIZACIÓN } \\
\hline 1 & $\begin{array}{l}\text { 1.-Distribución por sexo de la matrícula en enseñanzas artísticas } \\
\text { universitarias. } \\
\text { 1a.- Porcentaje de mujeres matriculadas en Bellas Artes. } \\
\text { 1b.- Porcentaje de mujeres matriculadas en Historia del Arte. } \\
\text { 1c.- Porcentaje de mujeres matriculadas en Estudios Superiores de Artes } \\
\text { Plásticas: Conservación y Restauración de Bienes Culturales. } \\
\text { 1d.- Porcentaje de mujeres matriculadas en Estudios Superiores de Artes } \\
\text { Plásticas: Diseño. }\end{array}$ \\
\hline 2 & $\begin{array}{l}\text { 2.-Distribución por sexos de la matrícula de enseñanzas artísticas no } \\
\text { universitarias. } \\
\text { 2a.- Porcentaje de mujeres matriculadas en FP -Grado Superior- Régimen } \\
\text { General: Artes Gráficas; Comunicación, Imagen y Sonido; Vidrio y } \\
\text { Cerámica. } \\
\text { 2b.- Porcentaje de mujeres matriculadas en FP -Grado Superior- Régimen } \\
\text { Especial Ciclos Formativos de Artes Plásticas. }\end{array}$ \\
\hline \multicolumn{2}{|c|}{ ACTIVIDAD } \\
\hline 3 & $\begin{array}{l}\text { 3.-Porcentaje de mujeres en las ocupaciones de Artes Visuales, según el Censo } \\
\text { de Población -2011- }\end{array}$ \\
\hline \multicolumn{2}{|c|}{ VISIBILIDAD-DIFUSIÓN } \\
\hline 4 & $\begin{array}{l}\text { 4.-Distribución por sexo de la obra expuesta en Museos y Centros de Arte. } \\
\text { 4a.- Porcentaje de obra femenina en las colecciones permanentes de } \\
\text { Museos y Centros de Arte. } \\
\text { 4b.- Exposiciones individuales de mujeres sobre el total de exposiciones } \\
\text { celebradas. } \\
\text { 4c.-Distribución por sexo de las exposiciones colectivas. }\end{array}$ \\
\hline
\end{tabular}




\begin{tabular}{|c|c|}
\hline Indicadores & Dimensión y descripción \\
\hline 5 & $\begin{array}{l}\text { 5.-Mujeres artistas representadas en la Feria ARCO. } \\
\text { 5a.- Porcentaje de mujeres en la dirección de ARCO. } \\
\text { 5b.- Porcentaje de mujeres artistas en ARCO. } \\
\text { 5c.- Porcentaje de mujeres artistas españolas en ARCO. }\end{array}$ \\
\hline 6 & $\begin{array}{l}\text { 6.-Mujeres galardonadas en los Premios Nacionales de Arte. } \\
\text { 6a.- Porcentaje de mujeres galardonadas en el PN Artes Plásticas. } \\
\text { 6b.- Porcentaje de mujeres galardonadas en el PN Restauración y } \\
\text { Conservación de Bienes Culturales. } \\
\text { 6c.- Porcentaje de mujeres galardonadas en el PN Fotografía. } \\
\text { 6d.- Porcentaje de mujeres galardonadas en el PN Velázquez. } \\
\text { 6e.- Porcentaje de mujeres galardonadas en el PN Moda. }\end{array}$ \\
\hline 7 & $\begin{array}{l}\text { 7.-Composición de los Jurados de los Premios Nacionales de Arte } \\
\text { 7a.- PN Artes Plásticas. } \\
\text { 7b.- PN Restauración y Conservación de Bienes Culturales. } \\
\text { 7c.- PN Fotografía. } \\
\text { 7d.- PN Velázquez. } \\
\text { 7e.- PN Moda. }\end{array}$ \\
\hline 8 & $\begin{array}{l}\text { 8.-Concesión de Becas } \\
\text { 8a.- Porcentaje de mujeres becadas en el programa FormARTE. } \\
\text { 8b.- Porcentaje de mujeres becadas en el programa Fullbright. } \\
\text { 8c.- Porcentaje de mujeres becadas en el programa Culturex. }\end{array}$ \\
\hline 9 & 9.- Obra expuesta en principales galerías. \\
\hline \multicolumn{2}{|c|}{ REDES PROFESIONALES } \\
\hline 10 & $\begin{array}{l}\text { 10a.- Adscripción de mujeres artistas en asociaciones de arte } \\
\text { 10b.- Presencia de mujeres en los órganos de decisión de asociaciones de } \\
\text { arte } \\
\text { 10c.- Puestos que ocupan las mujeres en Juntas Directivas }\end{array}$ \\
\hline
\end{tabular}




\begin{tabular}{|c|c|}
\hline Indicadores & Dimensión y descripción \\
\hline \multicolumn{2}{|l|}{ GESTIÓN } \\
\hline 11 & $\begin{array}{l}\text { 11a.- Directoras de Museos } \\
\text { 11b.- Mujeres galeristas } \\
\text { 11c.- Mujeres en Conserjerías autonómicas de cultura } \\
\text { 11d.- Mujeres en Comités de Redacción de Revistas de Arte }\end{array}$ \\
\hline
\end{tabular}

Fuente: Elaboración propia.

Tabla 6: Denominación completa de los Museos y Centros de Arte que conforman la muestra

\begin{tabular}{|l|l|l|}
\hline Centro & Denominación completa & Dirección \\
\hline CAAC & Centro Andaluz de Arte Contemporáneo & Varón \\
\hline CAC & Centro de Arte Contemporáneo de Málaga & Varón \\
\hline LABORAL & Centro de Arte y Creación Industrial & Varón \\
\hline ESBALUARD & Museu d'Art Modern i Contemporani de Palma & Varón \\
\hline TEA & Tenerife Espacio de las Artes & Varón \\
\hline CAAM & Centro Atlántico de Arte Moderno & Varón \\
\hline Da2 & Domus Artium & Varón \\
\hline MUSAC & Museo de Arte contemporáneo de Castilla y León & Varón \\
\hline MEV & Museo Estebán Vicente & Mujer \\
\hline PH & Museo de Arte Contemporáneo español Patio Herreriano & Mujer \\
\hline MACBA & Museu d'Art Contemporani de Barcelona & Varón \\
\hline IVAM & Instituto Valenciano de Arte Moderno & Mujer \\
\hline EACC & Espai d'Art Contemporani de Castelló & Varón \\
\hline MEIAC & Museo Extremeño e Iberoamericano de Arte Contemporáneo \\
\hline
\end{tabular}




\begin{tabular}{|l|l|l|}
\hline Centro & Denominación completa & Dirección \\
\hline MARCO & Museo de Arte Contemporánea de Vigo & Varón \\
\hline CGAV & Centro Galego de Arte Contemporánea & Varón \\
\hline MNCARS & Museo Nacional Centro de Arte Reina Sofía & Varón \\
\hline Thyssen & Museo de Arte Thyssen-Bornemisza & Varón \\
\hline CACH & Centro de Arte Contemporáneo Huarte & Varón \\
\hline Guggenheim & Museo Guggenheim Bilbao & Varón \\
\hline ARTIUM & Centro Museo Vasco de Arte Contemporáneo & Varón \\
\hline CCKM & Centro Cultural Koldo Mitxelena & Varón \\
\hline
\end{tabular}

Fuente: Website de los respectivos Centros de Arte. Elaboración propia, recuperado el $16 / \mathrm{X} / 2012$.

Tabla 7: Distribución por sexos de la composición de patronatos de museos

\begin{tabular}{|l|l|c|c|c|c|}
\hline \multirow{2}{*}{ CCAA } & \multirow{2}{*}{ Centro } & \multicolumn{2}{|c|}{ Presidencia } & \multicolumn{2}{|c|}{ Patronato } \\
\cline { 3 - 6 } & & Hombres & Mujeres & Hombres & Mujeres \\
\hline Asturias & Laboral & & $\mathrm{X}$ & 8 & 3 \\
\hline Baleares & Esbaluard & $\mathrm{X}$ & & 12 & 3 \\
\hline Canarias & CAAM & No disponible & 5 & 4 & \\
\hline \multirow{2}{*}{$\begin{array}{l}\text { Castilla y } \\
\text { León }\end{array}$} & Museo Esteban Vicente & $\mathrm{X}$ & & No disponible & \\
\cline { 2 - 6 } & Patio Herreriano & $\mathrm{X}$ & & 8 & 2 \\
\hline Cataluña & MACBA & $\mathrm{X}$ & & 28 & 9 \\
\hline \multirow{2}{*}{ Galicia } & MARCO & $\mathrm{X}$ & & 7 & 4 \\
\cline { 2 - 6 } & CGAC & $\mathrm{X}$ & & 20 & 4 \\
\hline
\end{tabular}




\begin{tabular}{|c|l|c|c|c|c|}
\hline \multirow{2}{*}{ CCAA } & \multirow{2}{*}{ Centro } & \multicolumn{2}{|c|}{ Presidencia } & \multicolumn{2}{|c|}{ Patronato } \\
\cline { 3 - 6 } & & Hombres & Mujeres & Hombres & Mujeres \\
\hline \multirow{2}{*}{ Madrid } & MNCARS & $\mathrm{X}$ & & 15 & 4 \\
\cline { 2 - 6 } & Thyssen & $\mathrm{X}$ & & 10 & 6 \\
\hline \multirow{2}{*}{ Navarra } & CACH & No disponible & 6 & 3 & \\
\hline \multirow{2}{*}{ País Vasco } & Guggenheim & $\mathrm{X}$ & & No disponible & \\
\cline { 2 - 6 } & Artium & $\mathrm{X}$ & & 11 & 5 \\
\hline TOTAL & & 1 & 130 & 47 & \\
\hline
\end{tabular}

Fuente: Website de los respectivos Centros de Arte. Recuperado el 20/III/2013. 